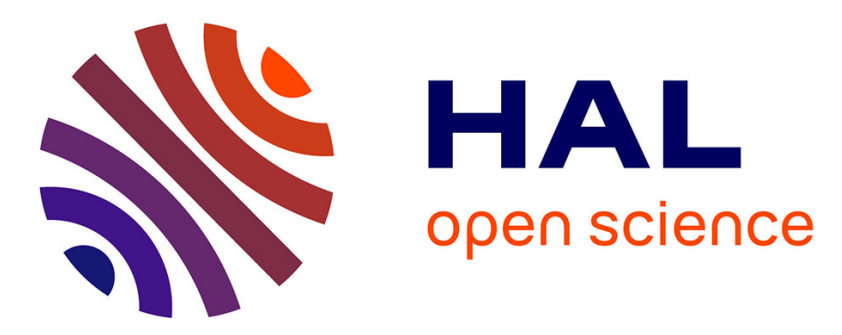

\title{
Absolute calibration of small angle neutron scattering data using strong coherent scattering
}

\author{
D. Lee, J. Barker, Si Chen
}

\section{To cite this version:}

D. Lee, J. Barker, Si Chen. Absolute calibration of small angle neutron scattering data using strong coherent scattering. Journal de Physique IV Proceedings, 1993, 03 (C8), pp.C8-431-C8-434. 10.1051/jp4:1993888 . jpa-00252319

\section{HAL Id: jpa-00252319 https://hal.science/jpa-00252319}

Submitted on 1 Jan 1993

HAL is a multi-disciplinary open access archive for the deposit and dissemination of scientific research documents, whether they are published or not. The documents may come from teaching and research institutions in France or abroad, or from public or private research centers.
L'archive ouverte pluridisciplinaire HAL, est destinée au dépôt et à la diffusion de documents scientifiques de niveau recherche, publiés ou non, émanant des établissements d'enseignement et de recherche français ou étrangers, des laboratoires publics ou privés. 


\title{
Absolute calibration of small angle neutron scattering data using strong coherent scattering
}

\author{
D.D. LEE, J. BARKER ${ }^{*}$ and S.H. CHEN
}

Massachusetts Institute of Technology, Cambridge, MA 02139, U.S.A.

${ }^{*}$ National Institute of Standards and Technology, Gaithersburg, MD 20899, U.S.A.

\begin{abstract}
Typically, small angle neutron scattering (SANS) data is normalized to an absolute scale using secondary standards such as water, polymers, silica gels, or irradiated aluminum. Errors for this method of calibration arise when the initial determination or calculation of the standard's scattering cross-section is no longer valid due to degradation or wavelength-dependent multiple scattering or detector efficiency effects. Here we illustrate how strong coherent scattering can be used to experimentally measure the incident neutron beam intensity and thus serve as a primary absolute calibration. We show how easily this procedure is implemented using a ternary microemulsion as a strong scatterer and compare its accuracy with some other secondary standards commonly in use. We find that the procedure is consistent with the other standards and should be considered a viable alternative when normalizing SANS measurements.
\end{abstract}

\section{Introduction}

As the number of instruments available to the general scientific community increases, small angle neutron scattering (SANS) is an ever more popular technique used to study structures and phenomena at distance scales from tens to thousands of Angstrom. A key aspect to utilizing the technique to its fullest extent involves normalizing data to an absolute scale. Absolute calibration allows the experimenter to calculate important structural parameters such as molecular weights, volume fractions, and specific surface areas [1], and to compare SANS results with other techniques like SAXS [2]. As a particular example of its usefulness, absolute SANS measurements led to the discovery of deuterium isotope effects in polystyrene-polybutadiene. block copolymers by Bates, Berney, Cohen and Wignall [3].

The differential scattering cross-section is defined as the scattered nentron intensity (particles $\mathrm{s}^{-1}$ ) per unit solid angle divided by the incident neutron flux (particles $\mathrm{cm}^{-2} \mathrm{~s}^{-1}$ ). Dividing this by the volume of the scatterer, one obtains the differential cross-section in units of $\mathrm{cm}^{-1}$ :

$$
\frac{d \Sigma}{d \Omega}(\theta)=\frac{1}{\bar{V}} \frac{I(\theta)}{\epsilon \Delta \bar{\Omega} T J_{0}}=\frac{1}{\epsilon I_{0}} \frac{I(\theta)}{d T \Delta \Omega}
$$

where $V$ is the volume of the scatterer, $d$ is its thickness, $I(\theta)$ is the neutron intensity measured by a detector pixel at scattering angle $\theta, \epsilon$ is the detection efficiency of the pixel, $\Delta \Omega$ is the solid angle subtended by the pixel, $T$ is the transmission of the scatterex, $J_{0}$ is the incident neutron flux, and $I_{0}=(V / d) \cdot J_{0}$ is the incident intensity. To eliminate beam intensity fluctuations, a second detector is normally used as a monitor and all measured intensities are typically normalized to some fixed number of monitor counts rather than to unit time. SANS results are also usually presented as functions of wave vector transfer $Q=\frac{4 \pi}{\lambda} \sin (\theta / 2)$ where $\lambda$ is the neutron wavelength.

In a typical SANS experiment, $T$ and $t(\theta)$ are experimentally measured, $d$ is known from the thickness of the sample cell, and $\Delta \Omega$ is easily calculated from the geometry of the instrument. Thus, in order to calculate the absolute differential cross-section, $\varepsilon I_{0}$ needs to be determined. Cotton [4] has ontlined two 
general methods which are used to determine this instrumental constant: measuring standards of known scattering cross-section and direct measurement of the incident neutron flux.

A wide variety of standards which are used at the National Center for Small-Angle Scattering Research (Oak Ridge National Laboratory) have previously been described in detail by Wignall and Bates [5]. The most accurate of these is the incoherent scattering from single-crystalline vanadium. However, due to its low counting rate and the inconvenience of repeating its annealing and etching, vanadium cannot be routinely used. Instead, secondary standards such as water, polymers, silica gels, and irradiated aluminum which have been previously calibrated using either vanadium or a beam fux measurement are employed.

The advantages for using such standards are obvious; their short measurement times and lack of any special preparation procedures enable users to quickly and conveniently calculate absolute calibrations. But the disadvantage with these secondary standards is that they are typically only accurate to about $10 \%$. Multiplescattering effects at larger wavelengths require determining empirical correction factors and complicate their use [6]. Wavelength-dependent detector efficiencies also make special correction factors necessary when using inelastic scattering standards such as water. The possibility of the standards themselves changing over time requires periodic calibration checks. All these factors contribute to the low accuracy of the computed absolute normalization constants.

The other calibration method involves a direct measurement of $I_{0}$ using a special high efficiency detector. Then the efficiency $\epsilon$ of the area detector is determined using precalibrated beam attenuators to avoid saturating and damaging it. Although in principle this procedure is very accurate, it is inconvenient for routine use since it involves setting up another detector. Also, subtle errors may arise from stacking precalibrated hydrogenous attenuators if backscattering from the attenuation causes an enhancement of the monitor count rate. We describe in detail here a method using a strong scatterer and the area detector to directly measure $\epsilon I_{0}$. A similar procedure using sintered graphite was mentioned before by Cotton [4] as being performed at the Laboratoire Léon Brillouin. Below, we show how easily this method is implemented using a ternary microemulsion as a strong scatterer and compare its accuracy with that obtained with some other standards.

\section{Experimental Method}

The procedure for using strong coherent scattering for absolute calibration is conceptually very simple. In Fig. 1 a schematic of a typical SANS instrument is shown. The first step of the procedure involves inserting an attenuator, removing the beam stop, and measuring the direct incident beam on the area detector. Next, a strong scattering sample is placed in the instrument and measured with and without the attenuator in order to calibrate it. From the sum of the counts on the area detector from the direct beam and the attenuation factor, $\epsilon I_{0}$ is easily computed.

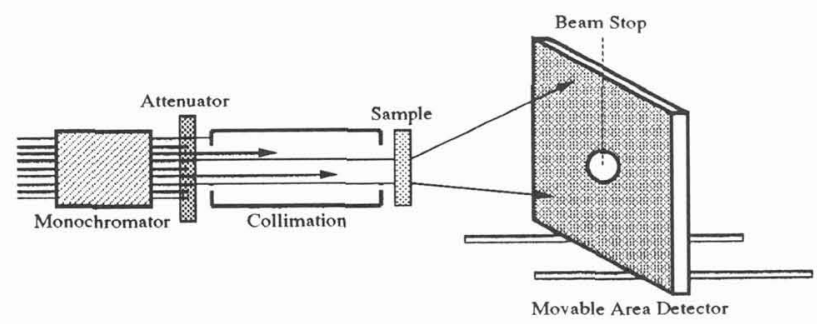

Figure 1: Schematic showing a typical SANS instrument setup.

In this study, acrylic slabs of different thicknesses which incoherently scattered away most of the incoming beam were used as attenuators. However, since acrylic's attenuation properties are very wavelengthdependent, it is only suitable for fixed-wavelength instruments. In a time-of-flight spectrometer where a broad range of neutron energies are utilized, wavelength-independent attenuation such as cadmium foil with small pinholes must be used.

There are several advantages for using a strong scatterer to calibrate the attenuator. First of all, it is very simple and convenient to implement. Short measurements times with and without the attenuator suffice to determine the attenuation constant and all the steps of the procedure are typically performed anyway while measuring transmissions. Since most of the scattering is coherent and elastic, wavelength-dependent detector efficiency effects can be safely ignored. Also, even though multiple scattering may be a significant portion of the total scattering, it does not affect the calculation of the attenuation factor. Thus, extremely strong scattering samples can be chosen to keep the calibration measuring time to a minimum. 
By distributing counts over the entire area detector, strong scatiering allows the atienuation factor to be more accurately determined than if only a few pixels were used. Different pixels record a wide range of counts which make saturation effects more readily apparent. Since the whole procedure can be repeated many times in the course of an experiment, the user can also easily check for self-consistency.

We illustrate this method using ternary microemulsion mixtures of tetraethylene glycol monodecyl ether $\left(\mathrm{C}_{10} \mathrm{E}_{4}\right)$, octane, and water at two different contrasts as strong scatterers. All measurements were performed at the Center for High Resolution Neutron Scattering 30 Meter SANS instrument at the National Institute of Standards and Technology. Incident nentrons were monochromated to a wavelength of $6 \AA(\Delta \lambda / \lambda=15 \%)$ using a multidisk mechanical velocity selector and collimated with pinholes 38 and $12 \mathrm{~mm}$ in diameters separated by a distance of $16.32 \mathrm{~m}$. After scattering from the sample, the neutrons were detected with a high data rate two-dimensional position sensitive detector $\left(65 \times 65 \mathrm{~cm}^{2}\right.$ with $1 \mathrm{~cm}$ resolution). The detector moved along rails which enabled the sample-to-detector distance to be quickly varied between 1.3 to 13 meters.

Fig. 2 shows a plot of the circularly averaged intensities from the first microemulsion scatterer at a sample-to-detector distance of 11.00 meters. After subtracting the dark count background, we see that the measurements with and without the attenuator are quite proportional. By matching the two curves, the attenuation factor of $.0102 \pm .0002$ is computed. This factor is then divided into the sum of the attenuated direct beam counts on the area detector to determine $\epsilon I_{0}$.

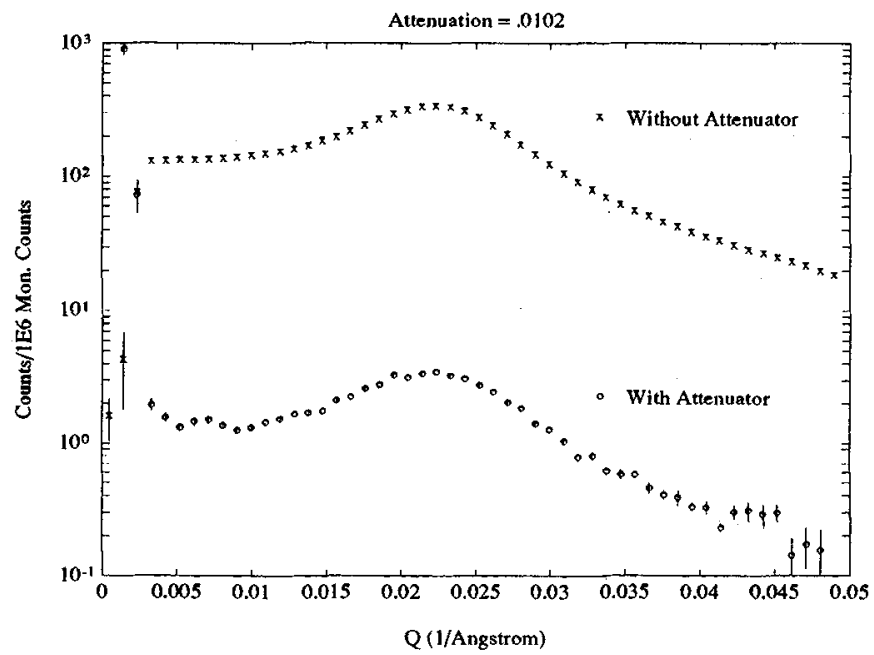

Figure 2: Circularly averaged data showing the microemulsion measured with and without an attenuator at a detector distance of 11.00 meters. By matching the two curves, the attenuation factor of .0102 was determined.

\section{Comparison with Other Standards}

All the standards mentioned below were corrected on a pixel to pixel basis for instrumental backgrounds, divided by the sample transmission and thickness, and normalized to a fixed number of monitor counts. The spatial variation of the detector efficiency was determined and corrected using the background-subtracted isotropic scattering from $\mathrm{H}_{2} \mathrm{O}$.

Table 1 shows the calibration constants as measured by the different standards. The two microemulsion samples were run at saniple-to-detector distances of 1.30 and 11.00 meters and were analyzed to obtain $\epsilon I_{0}$ as described above. The other standards are the secondary standards used on the SANS instruments at NIST. These standards were previously calibrated by directly measuring $I_{0}$ and the detector efficiency using a high efficiency $\mathrm{He}^{3}$ proportional counter. The standards were measured during the experiment and the scattering patterns obtained were fitted with known functional forms: a Guinier plot for the silica gel standard, the Random Phase Approximation equation for the binary polymer blends, and a constant for the water standard [7]. In addition, an empirical multiple scattering correction was applied to the polymer standards. Then the measured $d \Sigma / d \Omega(0)$ was calculated and compared with its previously calibrated value and $\epsilon I_{0}$ was deduced. 
The ertors for the NIST secondary standards are taken to be the quoted uncertainty level of $5 \%$ [ 7 ]. The main source of error for the absolute calibration using the microemulsions arises from the wavelengthdependent attenuation of acrylic. The attenuated beam will be slightly shifted toward lower wavelengths with an accompanying decrease in scattering from the microemulsions. For the wavelength and wavelength spread used in this study, we estimate this effect gives an uncertainty of about $3 \%$ to the calibration.

With the exception of water, the microemulsions and the other secondary standards agree to within their respective errors. Because of the large amount of inelastic scattering from the water, the calibration of the water standard varies with the wavelength dependence of the detector efficiency and consequently is very sensitive to the electronic configuration of the spectrometer such as preamplifier voltages and discriminator levels. We believe that small changes of these settings over time are responsible for the discrepancy in the water calibration.

\begin{tabular}{|l|r|r||}
\hline Standard & Det. Dist. $(\mathrm{m})$ & $\epsilon \bar{I}_{0}$ (per monitor) \\
\hline \hline Microemulsion 1 & 1.30 & $4.5 \pm .2$ \\
Microemulsion 2 & 1.30 & $4.4 \pm .2$ \\
$\mathrm{H}_{2}$ O & 1.30 & $5.3 \pm .6$ \\
\hline Microemulsion 1 & 11.00 & $4.3 \pm .1$ \\
Microemulsion 2 & 11.00 & $4.3 \pm .1$ \\
Silica Gel & 11.00 & $4.4 \pm .3$ \\
Polymer "B" & 11.00 & $4.6 \pm .3$ \\
Polymer "C" & 11.00 & $4.6 \pm .3$ \\
\hline
\end{tabular}

Table 1: Comparison between the various standards at different sample-detector distances.

\section{Conclusions}

We have shown that the calibration procedure using a strong scatterer is very easy to implement and consistent with the other standards. It appears that a more extensive study using various primary calibration techniques along with a wider range of neutron wavelengths and detector distances would be useful to show how applicable this procedure is under a variety of conditions. For the time being, this method should be considered a viable option when normalizing SANS data.

\section{Acknowledgements}

We would like to thank B. Orts for useful discussions on this topic. This material is based upon activities supported by the National Science Foundation under Agreement No. DMR-9122444 and the U, S. Department of Energy under Contract No. DE-FG02-90ER45429.

\section{References}

[1] G. Porod, in "Small Angle X-ray Scattering," eds. O. Glatter and O. Kratky, Academic Press, New York (1982).

[2] T. P. Russell, J. S. Lin, S. Spooner, and G. D. Wignall, J. Appl. Cryst. 21, 629-638 (1988).

[3] F. S. Bates, C. V. Berney, R. E Cohen, and G. D. Wignall, Polymer 24, 519-524 (1983).

[4] J. P. Cotton, in "Neutron, X-Ray and Light Scattering: Introduction to an Investigative Tool for Colloidal and Polymeric Systems," eds. P. Lindner and Th. Zemb, Elsevier, Amsterdam (1991).

[5] G. D. Wignall and F. S. Bates, J. Appl. Cryst. 20, 28-40 (1987).

[6] J. Schelten and W. Schmatz, J. Appl. Cryst. 13, 385-390 (1980).

[7] "SANS Data Reduction and Imaging Software," available at NIST (1993). 